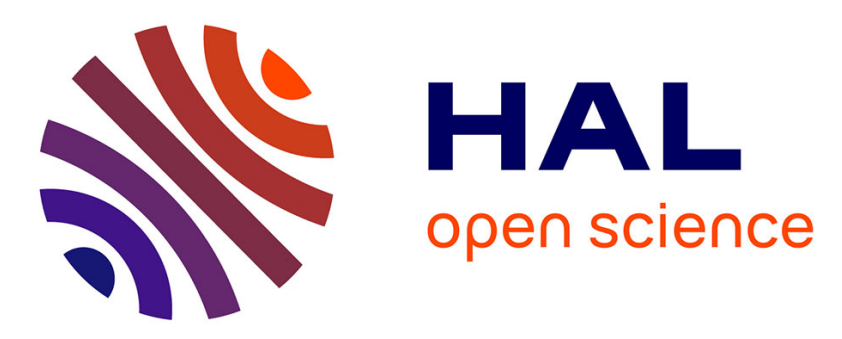

\title{
Amplitude-Dependent Internal Friction in Lead at Ambient Temperature
}

S. Kustov, S. Golyandin, K. Sapozhnikov, W. Robinson

\section{To cite this version:}

S. Kustov, S. Golyandin, K. Sapozhnikov, W. Robinson. Amplitude-Dependent Internal Friction in Lead at Ambient Temperature. Journal de Physique IV Proceedings, 1996, 06 (C8), pp.C8-265-C8268. 10.1051/jp4:1996856 . jpa-00254664

\section{HAL Id: jpa-00254664 https://hal.science/jpa-00254664}

Submitted on 1 Jan 1996

HAL is a multi-disciplinary open access archive for the deposit and dissemination of scientific research documents, whether they are published or not. The documents may come from teaching and research institutions in France or abroad, or from public or private research centers.
L'archive ouverte pluridisciplinaire HAL, est destinée au dépôt et à la diffusion de documents scientifiques de niveau recherche, publiés ou non, émanant des établissements d'enseignement et de recherche français ou étrangers, des laboratoires publics ou privés. 


\title{
Amplitude-Dependent Internal Friction in Lead at Ambient Temperature
}

\author{
S. Kustov, S. Golyandin, K. Sapozhnikov and W.H. Robinson* \\ A.F. Ioffe Physico-Technical Institute, Politekhnicheskaja 26, 194021 St. Petersburg, Russia \\ * Penguin Engineering Ltd (Pel), P.O. Box 33-093, Petone, New Zealand
}

\begin{abstract}
Amplitude-dependent internal friction of polycrystalline commercial lead has been studied in wide frequency and strain amplitude ranges. The strain amplitude dependence shows two stages characterized by different recovery times. These stages are attributed to different mechanisms: dislocation - point obstacle interaction at moderate amplitudes and interaction between dislocations along with their multiplication at the high ones. Pronounced and nonmonotonous dependence of the amplitude-dependent internal friction and elastic limit on prestrain is revealed. This peculiarity is attributed to transition from relatively homogeneous dislocation distribution to the inhomogeneous one due to formation of the cellular structure.
\end{abstract}

\section{INTRODUCTION}

Cyclic plasticity of lead is widely used in extrusion dampers and lead-rubber bearings for protecting constructions from earthquakes [1]. Fast recovery and recrystallisation of lead during and after deformation at ambient temperature assure an indefinite lifetime of lead based devices. The present work reports the results of investigation of damping properties, at different frequencies, and the recovery of lead, accompanying the prestrain at ambient temperatures in the range $0-30 \%$, by means of the internal friction techniques and observations of the microplastic strain.

\section{EXPERIMENTAL DETAILS}

Two experimental techniques were used for measurements. 1) A computer-controlled setup designed around piezoelectric ultrasonic composite oscillator technique allowed to study the amplitude-dependent internal friction (ADIF) and Young's modulus defect (YMD) at strain amplitudes $\sim\left(10^{-7}-3 * 10^{-4}\right)$ and frequency of about $100 \mathrm{kHz}$. It took approx. 5-10 min. (depending on number of points) to take an amplitude dependence of the decrement and resonant frequency, which normally included direct (strain amplitude increase) and reverse (strain amplitude decrease) runs. The oscillator was automatically tuned to a resonance at each strain amplitude. 2) Low-frequency computerized technique was based on the direct registration of stress - reversible anelastic strain hysteretic loops (HL) during cyclic loading of samples in compression by means of a hydraulic testing machine at room temperature and frequencies from 0.005 to $1 \mathrm{~Hz}$. A capacitance strain gauge with resolution of approx. $10^{-8}$ was used for strain measurements. Decrement $\delta$ was derived from the phase angle $\varphi$ between the first harmonic components of the applied stress and strain: $\delta=\pi \operatorname{tg} \varphi$. The samples were initially loaded to the stress, causing irreversible strain of the order of $10^{-4}$, in order to avoid accumulation of the irreversible strain during unipolar cyclic loading. After that the samples were unloaded to a fixed bias stress, on which an oscillatory sine wave stress was superimposed.

Samples of commercial polycrystalline lead of $99.97 \%$ purity, in a form of rectangular rods (approx. $1 * 1 * 6$ $\mathrm{mm}$ for the ultrasonic technique and $7 * 7 * 12 \mathrm{~mm}$ for the infrasonic one), were spark cut from a bar and annealed in vacuum for 2 hrs at $473 \mathrm{~K}$. Average grain size was initially approx. $2 \mathrm{~mm}$. To perform ultrasonic measurements immediately after prestrain (in less than $1 \mathrm{~min}$.), the samples were prestrained in compression along one of the short axes in a micrometric device on the length of approx. $5 \mathrm{~mm}$ consequently to different amounts of prestrain $(1,10$, and $30 \%$ ) without reglueing to an oscillator. 


\section{RESULTS AND DISCUSSION}

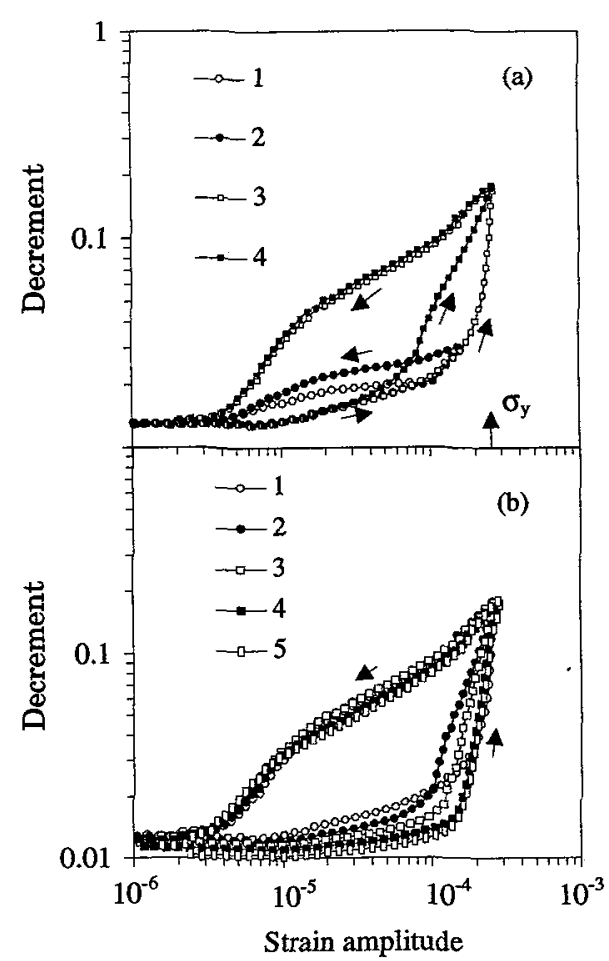

Figure 1: Strain amplitude dependences of the decrement for two annealed samples at room temperature and frequency of about $100 \mathrm{kHz}$, measured in consecutive runs: a) to maximum strain amplitude of $8^{*} 10^{-5}(1), 1.5^{*} 10^{-4}(2), 3.0^{*} 10^{-4}(3,4)$; b) to the same maximum strain amplitude with different time intervals between the measurements: 1 - the first measurement, $2-1 \mathrm{hr}$ after (1); $3-4 \mathrm{hrs}$ after (2); 4 - 24 hrs after (3); 5 - 72 hrs after (4). The arrow indicates the static yield stress $\sigma_{y}$.
Figure 1(a) shows the strain amplitude dependences of the decrement for an annealed sample, measured to consecutively increasing maximum strain amplitude. Amplitude hysteresis is persistent in all measurements, manifesting in higher decrement values on the reverse run, compared with the direct one. The amplitude hysteresis was essentially reversible for the measurements to maximum strain amplitudes below $\sim 10^{-4}$ (curves 1 and 2). Repeated direct runs are very close, indicating a very short time of the IF recovery, less than the time to measure the amplitude dependence. After exceeding a strain amplitude higher than $\sim 10^{-4}$, the direct runs of the amplitude dependences become very steep (curves 3 and 4 in Fig. 1(a)). The recovery of the IF during consecutive measurements is not complete in the highamplitude range (cf. curves 3 and 4). Slightly higher background decrement and more pronounced irreversible decrease of the resonant frequency of the sample are observed after the first measurement to a strain amplitude higher than $\sim 10^{-4}$. These changes are not revealed in the second run to the same strain amplitude. Figure 1 (b) depicts the results of the repeated ADIF measurements to the same maximum strain amplitude. Amplitude hysteresis at strain amplitudes higher than $\sim 10^{-4}$ is not reversible even after rather long time without high-amplitude excitation. In contrast to the high-amplitude range, low-amplitude damping may become even lower than in the initial annealed state after the highamplitude excitation. The high-amplitude range starts very close to the static yield stress. Dislocation multiplication appears to occur during the first ADIF measurement to a maximum strain amplitude higher than $\sim 10^{-4}$.

Results of consecutive measurements of the ADIF and YMD (resonant frequency) at $\mathrm{T}=6.5 \mathrm{~K}$ are displayed in Fig.2. The ADIF is two-staged like that at room temperature. "Normal" amplitude hysteresis is persistent in every measurement at moderate amplitudes, provided the maximum strain amplitude exceeds the one, attained in the previous measurement. By contrast, high-amplitude excitation is accompanied by an "anomalous" hysteresis (the ADIF on the reverse run is lower than on the direct one). Measurements below the maximum strain amplitudes, attained in previous measurements, coincide precisely with the reverse run of the measurement to the highest strain amplitude value, see curves 1 and 2 in Fig.2(b). This evidences for the stability of microstructure, produced by the previous measurement. The steep high amplitude stage is preserved to a great extent on the reverse and repeated runs. Therefore, dislocation multiplication process provides itself only a minor contribution to the ADIF.

Four samples were used to study the influence of prestrain on the ADIF of lead at $100 \mathrm{kHz}$. All samples exhibited qualitatively the same results. Figure 3(a) shows the amplitude dependences of the decrement of one of the samples in the annealed state and after different prestrains. The dependence of amplitude-dependent part of the total decrement on prestrain at two strain amplitudes, corresponding to the different ADIF stages, is shown for two samples in Fig.3(b). The ADIF is suppressed by prestrain up to $10 \%$ in both strain amplitude ranges. A significant increase of the decrement at $30 \%$ prestrain is observed.

Stress-strain curves and the examples of the HL, obtained during initial quasistatic loading of samples in the annealed state and after different prestrains are shown in Fig. 4. Irreversible strain is accumulated at the very initial stages of static loading of the samples. Following [2] we consider the stress, requined to produce a fixed (rather small, 

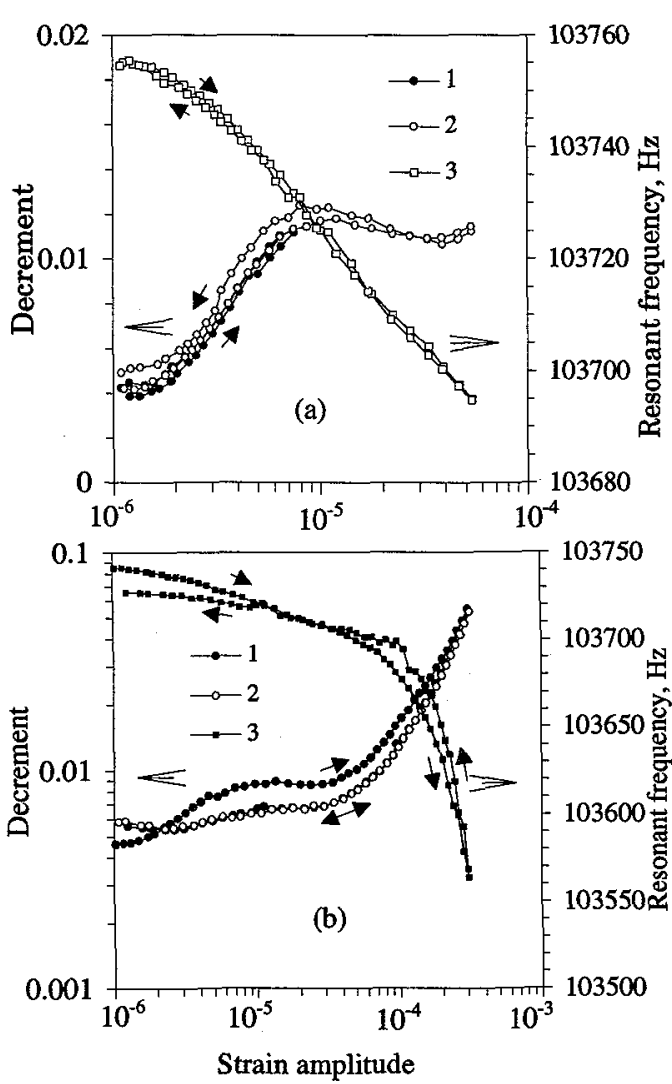

103750

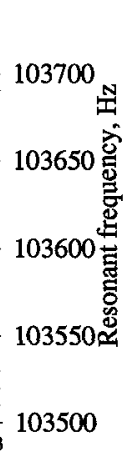

Figure 2: Strain amplitude dependences of the decrement of an annealed sample $(1,2)$ and resonant frequency of an oscillator (3) measured at temperature of $6.5 \mathrm{~K}$ in moderate (a) and high (b) strain amplitude ranges with increasing maximum strain amplitude: a) $8 * 10^{-6}$ (1) and $5 * 10^{-5}(2,3)$; b) $3 * 10^{-4}$ in the first $(1,3)$ and in the second (2) runs.

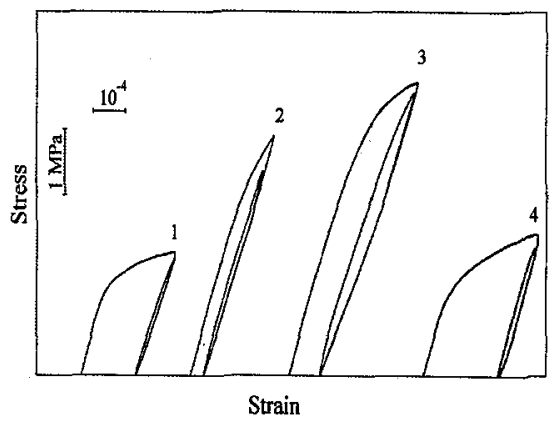

Figure 4: Initial loading of samples prestrained to 0 (1), $0.8(2), 10$ (3) and $30 \%$ (4) as measured by high-resolution capacity gauge. Closed stress-strain hysteretic loops were obtained during cyclic loading of the samples.
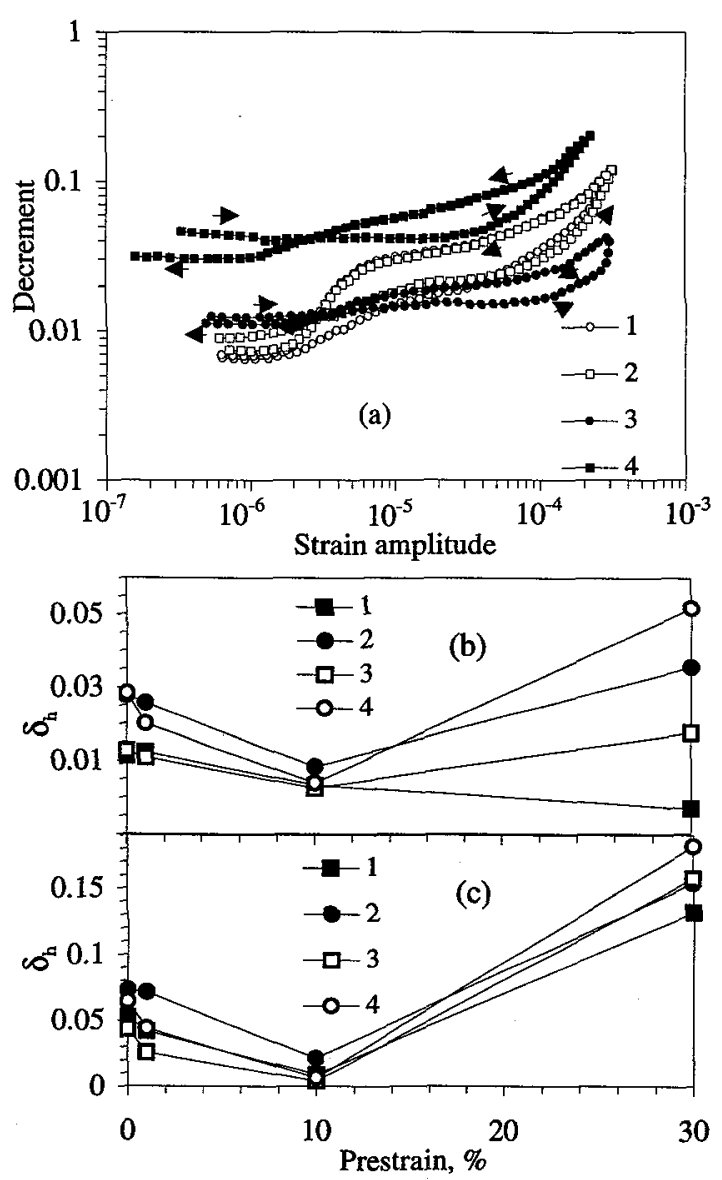

Figure 3: Influence of prestrain on the internal friction of lead at room temperature and frequency of about $100 \mathrm{kHz}$ : a) strain amplitude dependences of the decrement of a sample prestrained to 0 (1), 1 (2), 10 (3) and 30\% (4); $b, c)$ amplitude-dependent part of the decrement of two samples at strain amplitudes of $2^{*} 10^{-5}$ (b) and $2 * 10^{-4}$ (c) vs prestrain, obtained from the forward $(1,3)$ runs and reverse $(2,4)$ runs of strain amplitude dependences.

$\sim 10^{4}$ ) amount of plastic strain, as an elastic limit stress. Figure 4 indicates a strong increase of the elastic limit stress with prestrain up to $10 \%$. Further increase of the prestrain, on the contrary, decreases drastically the elastic limit stress. Thus, the prestrain has dramatic and nonmonotonous effect on the initial stage of stress-strain curve.

Figure 5 shows the ADIF measured at frequency 0.3 $\mathrm{Hz}$ by direct registration of the $\mathrm{HL}$. Maximum strain amplitude in each test corresponds approximately to the elastic limit. The ADIF is notable in the annealed sample, prestrained to 0.8 and $30 \%$, but is suppressed completely in the same strain amplitude range for the sample, prestrained the same strain amplitude range for the sample, prestrained to $10 \%$. The stage of the rapid increase in the ADIF with strain amplitude is not attained (curves
provided the anelastic strain during unipolar loading is reversible. The amplitude hysteresis is not 


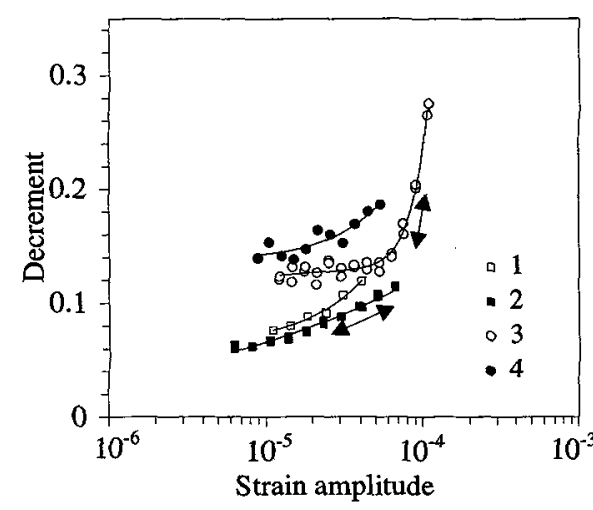

Figure 5: Strain amplitude dependences of the decrement, measured at frequency of $0.3 \mathrm{~Hz}$ by means of the direct registration of the stress-strain hysteretic loops for samples, prestrained to $0(1), 0.8$ (2), 10 (3) and 30\% (4).

revealed under these experimental conditions. Two-staged ADIF in lead for the same strain amplitude range was revealed in Ref. [3]. The stages were associated with the reversible and irreversible production of Frank-Read loops. Based on the data obtained, we suppose that different defect structure levels are responsible for the ADIF at low- and highamplitude stages. Dislocation interaction, accompanied by their multiplication, is responsible for the high-amplitude stage. The high-amplitude stage is not observed in infrasonic tests without accumulation of a considerable imeversible strain (Fig. 5). The lowamplitude ADIF is due to the dislocation - point obstacle interaction. High mobility of the point obstacles at room temperature gives rise to their redistribution by mobile dislocations, thus resulting in time dependence and amplitude hysteresis of the ADIF. The symmetric shape of the HL. (Fig. 4) rules out breakaway models for the ADIF. Thus, the ADIF behaviour at the low-amplitude stage may be ascribed to continuos pinningdepinning of mobile dislocations by mobile point obstacles. The "normal" amplitude hysteresis at $6.5 \mathrm{~K}$ is due to a tearing away of dislocations from the atmospheres, created during annealing. By all means, contact interaction of mobile dislocations with forest dislocations and their multiplication during high-amplitude excitation are accompanied by production of jogs on dislocations and vacancies in the bulk of a crystal. This leads to a decrease in average dislocation loop length and an increase in concentration of pinners and, therefore, to a decrease of dislocation mobility and ADIF ("anomalous" ADIF hysteresis). This explanation accounts also for the gradual decrease of the low-amplitude ADIF after the high-amplitude excitation (Fig. 1 (b)).

Both recovery and recrystallization are known to occur in lead at ambient termerature. Since grain size (for rather coarse grained lead) has no influence on the ADIF [3], recovery appears to be responsible for the rise in the ADIF after $30 \%$ prestrain. Following [2] we distinguish recovery (ortorecovery) and metarecovery. Recovery is considered as a general lowering of a stress-strain curve due to a thermal decrease of the net dislocation density. Metarecovery is a thermally-activated microstructure rearrangement, leading to a gradual initial part of a loading curve (decrease of the elastic limit). According to [2], it reflects a transition to a more heterogeneous (celullar) dislocation distribution in a bulk of a crystal. Influence of prestrain on micro- and macroplastic strain indicates strain hardening of lead and formation of relatively homogeneous dislocation structure at prestrains up to $10 \%$. This process is accompanied by the drastic suppress of the ADIF due to increase in dislocation density. Further increase of the prestrain leads to the formation of a cellular dislocation structure with low dislocation density within the cell's interior and, therefore, to higher ADIF. In a framework of nonlocalized friction approach, dislocation density controls both the high- and low-amplitude ADIF stages via the dislocation segment length between the nodes of a dislocation network. The most pronounced metarecovery is revealed at the highest prestrain. Therefore, metarecovery is enhanced by prestrain.

\section{CONCLUSIONS}

1) Two ADIF stages show different recovery times and correspond to different mechanisms:

- interaction of existing dislocations with point obstacles at moderate strain amplitudes,

- interaction of dislocations and their multiplication at strain amplitudes higher than $\sim 10^{-4}$.

2) Metarecovery of lead at ambient temperatures is enhanced by prestrain and manifests in ADIF at ultrasonic frequencies, low-frequency reversible microplastic strain and initial stage of macroplastic deformation. It is attributed to the formation of more heterogeneous (cellular) dislocation structure.

\section{References}

[1] Skinner R.I., Robinson W.H., and McVerry G.H., An Introduction to Seismic Isolation (Wiley, Chichester, 1993).

[2] Alden T.H., Met. Trans. 4 (1973) 1047-1057.

[3] Mason W.P., J.Acoust.Soc.Am. 28 (1956) 1207-1218. 